\title{
Stressed hearts in children with obesity and diabetes: a cause for concern?
}

\author{
C. Berry • N. Sattar
}

Received: 3 December 2010 /Accepted: 14 December 2010/Published online: 19 January 2011

(C) Springer-Verlag 2011

\begin{abstract}
Obesity in young people is an emerging public health problem, particularly because of its association with type 2 diabetes. Since obesity and diabetes contribute to the development of cardiovascular disease in adults, the question arises as to whether or not these conditions may be associated with cardiovascular abnormalities in children and adolescents. In this issue of Diabetologia, Shah et al. report the results of a cross-sectional study of heart structure and function in 612 adolescents and young adults (aged 10-24 years) subdivided into three groups: (1) those with obesity and type 2 diabetes; (2) those with type 2 diabetes but without obesity; and (3) lean healthy controls. Their results revealed that left ventricular mass (indexed to body surface area) was greater in the obese individuals than in lean controls. Left ventricular systolic function was more dynamic in obese participants and obese participants with type 2 diabetes compared with lean controls, whereas systolic function was comparable in obese patients with or without type 2 diabetes. Furthermore, compared with the healthy lean control participants, diastolic function was impaired in the obese group and further impaired in the obese individuals with diabetes. These results, and those of a few other similar studies, lend support to the notion that obesity and diabetes in children cause subtle abnormalities in cardiovascular structure and function. The present
\end{abstract}

C. Berry $(\bowtie) \cdot$ N. Sattar

Institute of Cardiovascular and Medical Sciences,

BHF Glasgow Cardiovascular Research Centre,

126 University Place, University of Glasgow,

Glasgow G12 8TA, UK

e-mail: colin.berry@glasgow.ac.uk

C. Berry

Golden Jubilee National Hospital,

Glasgow, UK commentary discusses potential mechanisms and possible clinical ramifications for such findings.

\section{Introduction}

Obesity is a major public health problem, particularly because of its association with type 2 diabetes and its associated cardiovascular consequences [1]. Obesity in adult life confers an increased risk of a broad range of cardiovascular health problems including hypertension, coronary artery disease, heart failure, pulmonary embolism and stroke $[1,2]$. Obesity in children and adolescents is a major concern because premature weight gain in youth might establish an enhanced risk of premature cardiovascular health problems in adulthood. This possibility raises the question of whether or not obesity in young people might adversely affect cardiovascular health even before adulthood. In other words, might there be measurable cardiovascular abnormalities in overweight and obese young people? Furthermore, as more and more young people develop type 2 diabetes, is there any evidence that early onset diabetes accelerates obesity-related cardiovascular effects?

In adults, an increase in fat mass predisposes to several changes in the cardiovascular system, including an increase in vascular volume, an increased cardiac output and an increase in systemic vascular resistance. The long-term consequences of these changes may include high blood pressure and left ventricular hypertrophy, which may manifest even in young people [3]. In adults, chronic obesity can affect the pulmonary circulation in a number of ways, including chronic hypoventilation, sleep apnoea and possibly thromboembolic disease, all of which may lead to pulmonary vascular remodelling and pulmonary hypertension. Whether or not alterations in cardiovascular structure 
and function might also occur in overweight children is less well established.

Of course, the underlying reasons for weight gain in children are very relevant to cardiovascular health. The early causes of obesity in young people are multifactorial [4], and include genetic factors, intrauterine factors (especially diabetes during pregnancy), and rapid infant weight gain (especially environmental factors, including short duration of breastfeeding) [5, 6]. Environmental and lifestyle factors leading to overeating and lowered physical activity are probably most important, especially in adolescence $[6,7]$; in turn, obesity almost certainly contributes to reduced physical activity in children. Irrespective of cause, obesity in children is linked to insulin resistance and a number of other metabolic changes [7], but, as yet, their direct and indirect links with vascular morphology or function remain to be fully established. Similarly, obesity and raised blood pressure are closely linked $[1,2]$ and it would be interesting to know whether increased left ventricular mass in overweight young people is in fact related predominantly to the effects of high blood pressure, or whether other obesity-related perturbations also play a role.

\section{Heart structure and function in obese young adults}

Heart disease in children is usually congenital and, in this sense, obesity is not associated with symptomatic heart disease in young people. However, whether or not obesity may cause subtle or subclinical impairments in cardiovascular function in children is a subject of current interest. Some studies suggest that obesity in young people is associated with pathological changes consistent with cardiovascular disease, such as albuminuria [8], increased circulating concentrations of natriuretic peptides and carotid intimal thickening $[9,10]$.

Heart structure and function can be measured in a number of different ways both invasively and non-invasively. Noninvasive methods, such as echocardiography and magnetic resonance imaging, can provide accurate assessments of heart geometry, mass and function. Alternatively, invasive intravascular methods, such as transcatheter measurement of ventricular pressure and volume, provide 'gold standard' quantification of left and right ventricular systolic and diastolic function. However, as echocardiography is safe and well tolerated, brief and relatively inexpensive, this method is generally preferred at least for the initial assessment of heart structure and function [11].

Heart pump function is a complex cyclical process of contraction and relaxation. In systole, ventricular contraction involves muscle fibre shortening and counter-clockwise twisting, particularly at the apex; in diastole, the myocardium relaxes dynamically with expansion of the ventricles, which subsequently fill with blood through passive and active filling from the atria. Conditions that adversely affect heart function may act through alterations in afterload (i.e. arterial hypertension leading to increased systemic vascular resistance) or preload (i.e. reduced or increased ventricular filling pressures), or more directly by affecting myocardial function. Clearly, systolic function is important as heart pump function drives arterial blood through the circulatory system. However, diastole is just as important to ensure adequate ventricular filling which, according to the Frank-Starling law of the heart, is a determinant of cardiac output. This means that both systolic and diastolic functions are interdependent and, wherever possible, should be evaluated together in studies of heart function [11]. In adults, obesity and diabetes are associated with subtle impairments in systolic and diastolic function, but whether or not these problems may occur earlier in life is uncertain.

\section{Relationships between body fat, insulin resistance and alterations in heart structure and function in young people}

Investigators at the Cincinnati Children's Hospital Medical Centre (OH, USA), have been studying the cardiovascular effects of obesity in children for several years. In this issue of Diabetologia, Shah et al. [12] report the results of a cross-sectional study of heart structure and function in 612 adolescents and young adults (aged 10-24 years) subdivided into three groups: (1) those with obesity and type 2 diabetes; (2) those with type 2 diabetes but without obesity; and (3) lean healthy controls. The diagnosis of type 2 diabetes was based on the American Diabetes Association criteria. All but one of the participants with diabetes was non-insulin requiring. Shah et al. [12] found a number of differences in heart structure and function between these groups. First, heart geometry differed between the groups and left ventricular mass (indexed to body surface area) was greater in the obese individuals than in lean controls. Left ventricular systolic function was more dynamic in obese participants and obese participants with type 2 diabetes compared with lean controls, whereas systolic function was comparable in obese patients with or without type 2 diabetes.

Some more striking findings were apparent for diastolic function, which is the phase of the cardiac cycle after systole when ventricular filling occurs. Compared with the healthy lean control participants, diastolic function was impaired in the obese group and further impaired in the obese individuals with diabetes. Impairments in diastolic function are associated with symptoms such as premature breathlessness with exercise, leading in turn to exercise intolerance. In fact, diastolic dysfunction (or impairments in 
ventricular filling and relaxation) is not only associated with left ventricular hypertrophy but diastolic dysfunction may lead to symptoms and signs of heart failure [11]. Taken together, as Shah et al. [12] have shown alterations in heart structure and function in obese adolescents and young adults, their observations suggest that insulin resistance and/or hyperglycaemia may enhance the effects of obesity on the heart, a worrying observation as the obesity epidemic is clearly driving down the average age of diagnosis of type 2 diabetes, with a substantial rise in the number of cases in the second to fourth decades of life.

Are the results in this paper sufficiently comprehensive? Probably not. While the study by Shah et al. [12] involved a large number of reasonably well-characterised individuals it does not provide answers on the natural history of how weight gain and insulin resistance (or its associated metabolic abnormalities) might affect heart structure and function. The authors admit that as their study involved only a single cross-sectional assessment it is difficult to draw causal relationships between obesity, diabetes and altered cardiac structure and function. Recent studies by Schuster et al. [13] have found that in overweight prepubertal boys, abnormalities in left ventricular diastolic function are related to the severity of obesity and signs of diastolic dysfunction become more pronounced with exercise. Future studies in this area should involve serial assessments over time in order to work out the temporal relationships between metabolic abnormalities and changes in the heart.

It is equally important to question whether the measurable changes in heart geometry and function associated with obesity and diabetes in young people are permanent or reversible. Clinical studies with interventions that selectively reduce either obesity or insulin resistance (or commonly both) would be needed to answer this question. Clearly, exercise is an attractive intervention that would seem particularly appropriate for young people who have sedentary lifestyles. However, to date, we are not aware of any study that has convincingly shown that an intervention based on physical activity alone reduces bodyweight in obese young people. More research is needed in this area to help clarify outstanding questions. For example, might an exercise-based intervention have beneficial effects on heart structure and function in obese young people with measurable cardiac abnormalities and, if so, what might be the mechanisms: weight reduction, improvements in insulin resistance and/or both? A change in diet to lessen habitual energy intake in a sustainable manner is of course another pragmatic intervention for overweight young people, and there is some evidence that dietary intervention alone can have benefits for heart function [14, 15].

How clinically relevant are the cardiac changes in the present study? The difference in left ventricular mass observed between obese individuals (with or without diabetes) and lean participants (about a 40\% increase) and alterations in left ventricular geometry (remodelling) were quite marked. With respect to ventricular pump function, there were subtle differences in systolic function at rest and more pronounced differences in diastolic function. While these abnormalities are unlikely to compromise activities of daily living (particularly for those with a sedentary lifestyle), one might anticipate exercise intolerance with effort [13], an important consideration since obesity may precede declining activity levels in some (perhaps many) children. Chronically morbidly obese children may be at risk of premature cardiovascular disease, but epidemiological studies (and case reports) will be needed to help confirm or refute whether cardiovascular disease might actually occur in young people.

In order to dig deeper into these questions, future studies should involve cardiorespiratory (i.e. cardiometabolic) exercise testing, ideally with serial assessments over time. Cardiorespiratory exercise testing is a standard method to quantify cardiorespiratory fitness with measurement of oxygen consumption ( $\dot{V} \mathrm{O}_{2 \max }$, a measure of cardiorespiratory fitness) and other indices such as anaerobic threshold. The US National Health and Nutrition Examination Survey (NHANES, 1999-2002) included a standard submaximal exercise tolerance test in 1,078 adolescents aged 12-18 years, together with assessments of obesity, waist circumference and insulin resistance (HOMA) [16]. There was an inverse relationship between $\dot{V} O_{2 \text { peak }}$ and body weight and a positive association with lean body (muscle) mass, as revealed by dual-emission X-ray absorptiometry. Furthermore, insulin resistance correlated inversely with $\dot{V} O_{2 \text { peak }}$ in boys but not girls and, overall, the inverse relationship between insulin resistance and oxygen utilisation was mainly apparent in obese boys [16]. This is an impressive study because of the large number of participants who underwent exercise testing; however, as the authors acknowledge, the study assessments took place at a single time point and did not involve 'full' or maximal exercise testing.

Finally, does body fat distribution influence heart structure and function independently of fat mass? As in adulthood, waist circumference in young people is positively related to left ventricular mass and echocardiographic measures of diastolic function, independent of body mass index [17]. Beyond central adiposity, epicardial fat distribution appears to be associated with deleterious effects on heart structure and function [18]. While these observations are of interest, it should be noted that recent data from a much larger prospective study in children and adolescents suggest measurements of waist circumference or directly assessed fat mass in childhood do not appear to be associated with cardiovascular risk factors (lipids, insulin or blood pressure) in adolescence any more strongly than 
BMI [19]. Clearly, large comprehensive prospective studies of cardiac function are needed to help us answer some of the outstanding questions raised herein, including the relevance of body fat distribution over BMI.

\section{Conclusion}

In conclusion, emerging research confirms that obesity and type 2 diabetes in young people predispose to reduced levels of activity and abnormalities in cardiovascular structure and function. These findings should draw further attention to the need for public health interventions to prevent and treat obesity and type 2 diabetes in young people.

Acknowledgements C. Berry is supported by a Senior Clinical Fellowship from the Scottish Funding Council. His research is also supported by the British Heart Foundation, Medical Research Scotland and the Chief Scientist Office of the Scottish Health Department. N. Sattar's research is supportad by the British Heart Foundation, the Chief Scientist Office of the Scottish Health Department, Diabetes UK, the National Institute for Health Research, the Medical Research Council and Wellcome Trust.

Duality of interest The authors declare that there is no duality of interest associated with this manuscript.

\section{References}

1. Lawlor DA, Lean M, Sattar N (2006) ABC of obesity: obesity and vascular disease. BMJ 333:1060-1063

2. Guh DP, Zhang W, Bansback N, Amarsi Z, Birmingham CL, Anis AH (2009) The incidence of co-morbidities related to obesity and overweight: a systematic review and meta-analysis. BMC Public Health 25(9):88

3. Dhuper S, Abdullah RA, Weichbrod L, Mahdi E, Cohen HW (2011) Association of obesity and hypertension with left ventricular geometry and function in children and adolescents. Obesity $19: 128-133$

4. Ong KK (2010) Early determinants of obesity. Endocr Dev 19:53-61

5. Lamb MM, Dabelea D, Yin X et al (2010) Early-life predictors of higher body mass index in healthy children. Ann Nutr Metab 56:16-22

6. Graff M, North KE, Monda KL et al. (2010) The combined influence of genetic factors and sedentary activity on body mass changes from adolescence to young adulthood: the National Longitudinal Adolescent Health Study. Diabetes Metab Res Rev. doi:10.1002/dmrr.1147
7. Owen CG, Nightingale CM, Rudnicka AR et al (2010) Physical activity, obesity and cardiometabolic risk factors in 9- to 10-yearold UK children of white European, South Asian and black African-Caribbean origin: the Child Heart And health Study in England (CHASE). Diabetologia 53:1620-1630

8. Hirschler V, Molinari C, Maccallini G, Aranda C (2010) Comparison of various maternal anthropometric indices of obesity for identifying metabolic syndrome in offspring. Pediatr Diabetes $11: 322-330$

9. Urbina EM, Kimball TR, McCoy CE, Khoury PR, Daniels SR, Dolan LM (2009) Youth with obesity and obesity-related type 2 diabetes mellitus demonstrate abnormalities in carotid structure and function. Circulation 119:2913-2919

10. Saritas T, Tascilar E, Abaci A et al (2010) N-terminal BNP, epicardial obesity and carotid intima thickness in asymptomatic obese children. Pediatr Cardiol 31:792-799

11. Task Force for Diagnosis and Treatment of Acute and Chronic Heart Failure 2008 of European Society of Cardiology, Dickstein K, Cohen-Solal A et al (2008) ESC guidelines for the diagnosis and treatment of acute and chronic heart failure 2008: the Task Force for the Diagnosis and Treatment of Acute and Chronic Heart Failure 2008 of the European Society of Cardiology. Developed in collaboration with the Heart Failure Association of the ESC (HFA) and endorsed by the European Society of Intensive Care Medicine (ESICM). Eur Heart J 29:2388-2442

12. Shah AS, Khoury PR, Dolan LM et al. (2011) The effects of obesity and type 2 diabetes mellitus on cardiac structure and function in adolescents and young adults. Diabetologia. doi:10.1007/s00125-010-1974-7

13. Schuster I, Karpoff L, Perez-Martin A et al (2009) Cardiac function during exercise in obese prepubertal boys: effect of degree of obesity. Obesity 17:1878-1883

14. Zeybek C, Celebi A, Aktuglu-Zeybek C et al (2010) The effect of low-carbohydrate diet on left ventricular diastolic function in obese children. Pediatr Int 52:218-223

15. Zeybek C, Aktuglu-Zeybek C, Onal H, Altay S, Erdem A, Celebi A (2009) Right ventricular subclinical diastolic dysfunction in obese children: the effect of weight reduction with a lowcarbohydrate diet. Pediatr Cardiol 30:946-953

16. Cummings DM, Dubose KD, Imai S, Collier DN (2010) Fitness vs fatness and insulin resistance in U.S. adolescents. J Obes pii: 195729

17. Mehta SK, Richards N, Lorber R, Rosenthal GL (2009) Abdominal obesity, waist circumference, body mass index, and echocardiographic measures in children and adolescents. Congenit Heart Dis 4:338-347

18. Ozdemir O, Hizli S, Abaci A, Agladioglu K, Aksoy S (2010) Echocardiographic measurement of epicardial adipose tissue in obese children. Pediatr Cardiol 31:853-860

19. Lawlor DA, Benfield L, Logue J et al (2010) Association between general and central adiposity in childhood, and change in these, with cardiovascular risk factors in adolescence: prospective cohort study. BMJ 341:c6224 